\title{
Position dependent random maps in one and higher dimensions
}

\author{
by \\ Wael Bahsoun (Victoria) and PaWę Góra (Montreal)
}

\begin{abstract}
A random map is a discrete-time dynamical system in which one of a number of transformations is randomly selected and applied on each iteration of the process. We study random maps with position dependent probabilities on the interval and on a bounded domain of $\mathbb{R}^{n}$. Sufficient conditions for the existence of an absolutely continuous invariant measure for a random map with position dependent probabilities on the interval and on a bounded domain of $\mathbb{R}^{n}$ are the main results.
\end{abstract}

1. Introduction. Let $\tau_{1}, \ldots, \tau_{K}$ be a collection of transformations from $X$ to $X$. Usually, the random map $T$ is defined by choosing $\tau_{k}$ with constant probability $p_{k}, p_{k}>0, \sum_{k=1}^{K} p_{k}=1$. The ergodic theory of such dynamical systems was studied in [9] and in [8] (see also [7]).

There is a rich literature on random maps with position dependent probabilities with $\tau_{1}, \ldots, \tau_{K}$ being continuous contracting transformations (see [10]).

In this paper, we deal with piecewise monotone transformations $\tau_{1}, \ldots, \tau_{K}$ and position dependent probabilities $p_{k}(x), k=1, \ldots, K$, where $p_{k}(x)>0$, $\sum_{k=1}^{K} p_{k}(x)=1$, i.e., the $p_{k}$ 's are functions of position. We point out that studying such dynamical systems was begun in [5], where sufficient conditions for the existence of an absolutely continuous invariant measure were given. The conditions in [5] are applicable only when $\tau_{1}, \ldots, \tau_{K}$ are $C^{2}$ expanding transformations (see [5] for details). In this paper, we prove the existence of an absolutely continuous invariant measure for a random map $T$ on $[a, b]$ under milder conditions (see Section 4, Conditions (A) and (B)). Moreover, we prove the existence of an absolutely continuous invariant measure for a random map $T$ on a bounded domain of $\mathbb{R}^{n}$ (see Section 6 , Condition (C)).

2000 Mathematics Subject Classification: Primary 37A05, 37E05.

Key words and phrases: random map, absolutely continuous invariant measure, Perron-Frobenius operator.

The research of P.G. was supported by NSERC grant. W.B. is a recipient of PIMS postdoctoral fellowship. 
The paper is organized in the following way: In Section 2, following the ideas of [5], we formulate the definition of a random map $T$ with position dependent probabilities and introduce its Perron-Frobenius operator. In Section 3, we prove some properties of the Perron-Frobenius operator of $T$. In Section 4, we prove the existence of an absolutely continuous invariant measure for $T$ on $[a, b]$. In Section 5 , we give an example of a random map $T$ which does not satisfy the conditions of [5]; yet, it preserves an absolutely continuous invariant measure under conditions (A) and (B). In Section 6, we prove the existence of an absolutely continuous invariant measure for $T$ on a bounded domain of $\mathbb{R}^{n}$. In Section 7, we give an example of a random map in $\mathbb{R}^{n}$ that preserves an absolutely continuous invariant measure.

2. Preliminaries. Let $(X, \mathfrak{B}, \lambda)$ be a measure space, where $\lambda$ is an underlying measure. Let $\tau_{k}: X \rightarrow X, k=1, \ldots, K$, be piecewise oneto-one, non-singular transformations on a common partition $\mathcal{P}$ of $X: \mathcal{P}=$ $\left\{I_{1}, \ldots, I_{q}\right\}$ and $\tau_{k, i}=\left.\tau_{k}\right|_{I_{i}}, i=1, \ldots, q, k=1, \ldots, K(\mathcal{P}$ can be found by considering finer partitions). We define the transition function for the random map $T=\left\{\tau_{1}, \ldots \tau_{K} ; p_{1}(x), \ldots, p_{K}(x)\right\}$ as follows:

$$
\mathbb{P}(x, A)=\sum_{k=1}^{K} p_{k}(x) \chi_{A}\left(\tau_{k}(x)\right),
$$

where $A$ is any measurable set and $\left\{p_{k}(x)\right\}_{k=1}^{K}$ is a set of position dependent measurable probabilities, i.e., $\sum_{k=1}^{K} p_{k}(x)=1, p_{k}(x) \geq 0$ for any $x \in X$, and $\chi_{A}$ denotes the characteristic function of the set $A$. We define $T(x)=\tau_{k}(x)$ with probability $p_{k}(x)$ and $T^{N}(x)=\tau_{k_{N}} \circ \tau_{k_{N-1}} \circ \cdots \circ \tau_{k_{1}}(x)$ with probability $p_{k_{N}}\left(\tau_{k_{N-1}} \circ \cdots \circ \tau_{k_{1}}(x)\right) \cdot p_{k_{N-1}}\left(\tau_{k_{N-2}} \circ \cdots \circ \tau_{k_{1}}(x)\right) \cdots p_{k_{1}}(x)$. The transition function $\mathbb{P}$ induces an operator $\mathbb{P}_{*}$ on measures on $(X, \mathfrak{B})$ defined by

$$
\begin{aligned}
\mathbb{P}_{*} \mu(A) & =\int \mathbb{P}(x, A) d \mu(x)=\sum_{k=1}^{K} \int_{k}(x) \chi_{A}\left(\tau_{k}(x)\right) d \mu(x) \\
& =\sum_{k=1}^{K} \int_{\tau_{k}^{-1}(A)} p_{k}(x) d \mu(x)=\sum_{k=1}^{K} \sum_{i=1}^{q} \int_{\tau_{k, i}^{-1}(A)} p_{k}(x) d \mu(x) .
\end{aligned}
$$

We say that the measure $\mu$ is $T$-invariant iff $\mathbb{P}_{*} \mu=\mu$, i.e.,

$$
\mu(A)=\sum_{k=1}^{K} \int_{\tau_{k}^{-1}(A)} p_{k}(x) d \mu(x), \quad A \in \mathfrak{B} .
$$

If $\mu$ has density $f$ with respect to $\lambda$, then $\mathbb{P}_{*} \mu$ also has a density which we denote by $P_{T} f$. By change of variables, we obtain 


$$
\begin{aligned}
\int_{A} P_{T} f(x) d \lambda(x) & =\sum_{k=1}^{K} \sum_{i=1}^{q} \int_{\tau_{k, i}^{-1}(A)} p_{k}(x) f(x) d \lambda(x) \\
& =\sum_{k=1}^{K} \sum_{i=1}^{q} \int_{A} p_{k}\left(\tau_{k, i}^{-1} x\right) f\left(\tau_{k, i}^{-1} x\right) \frac{1}{J_{k, i}\left(\tau_{k, i}^{-1}\right)} d \lambda(x),
\end{aligned}
$$

where $J_{k, i}$ is the Jacobian of $\tau_{k, i}$ with respect to $\lambda$. Since this holds for any measurable set $A$ we obtain an a.e. equality

$$
\left(P_{T} f\right)(x)=\sum_{k=1}^{K} \sum_{i=1}^{q} p_{k}\left(\tau_{k, i}^{-1} x\right) f\left(\tau_{k, i}^{-1} x\right) \frac{1}{J_{k, i}\left(\tau_{k, i}^{-1}\right)} \chi_{\tau_{k}\left(I_{i}\right)}(x)
$$

or

$$
\left(P_{T} f\right)(x)=\sum_{k=1}^{K} P_{\tau_{k}}\left(p_{k} f\right)(x)
$$

where $P_{\tau_{k}}$ is the Perron-Frobenius operator corresponding to the transformation $\tau_{k}$ (see [1] for details). We call $P_{T}$ the Perron-Frobenius operator of the random map $T$. It is main tool in this paper, with very useful properties.

3. Properties of the Perron-Frobenius operator of $T$. The properties of $P_{T}$ resemble the properties of the classical Perron-Frobenius operator of a single transformation.

LEMMA 3.1. $P_{T}$ has the following properties:

(i) $P_{T}$ is linear;

(ii) $P_{T}$ is non-negative; i.e., $f \geq 0 \Rightarrow P_{T} f \geq 0$;

(iii) $P_{T} f=f \Leftrightarrow \mu=f \cdot \lambda$ is T-invariant;

(iv) $\left\|P_{T} f\right\|_{1} \leq\|f\|_{1}$, where $\|\cdot\|_{1}$ denotes the $L^{1}$ norm;

(v) $P_{T \circ R}=P_{R} \circ P_{T}$. In particular, $P_{T}^{N} f=P_{T^{N}} f$.

Proof. The proofs of (i)-(iv) are analogous to those for a single transformation. For the proof of (v), let $T$ and $R$ be two random maps corresponding to $\left\{\tau_{1}, \ldots, \tau_{K} ; p_{1}, \ldots, p_{K}\right\}$ and $\left\{\zeta_{1}, \ldots, \zeta_{L} ; r_{1}, \ldots, r_{L}\right\}$ respectively. We define $\left\{\tau_{k}\right\}_{k=1}^{K}$ and $\left\{\zeta_{l}\right\}_{l=1}^{L}$ on a common partition $\mathcal{P}$. We have

$$
\begin{aligned}
P_{R}\left(P_{T} f\right) & =P_{R}\left(\sum_{k=1}^{K} P_{\tau_{k}}\left(p_{k} f\right)\right) \\
& =\sum_{l=1}^{L} \sum_{k=1}^{K} P_{\zeta_{l}}\left(r_{l} P_{\tau_{k}}\left(p_{k} f\right)\right) \\
& =\sum_{l=1}^{L} \sum_{k=1}^{K} \sum_{i=1}^{q} r_{l}\left(\zeta_{l, i}^{-1}\right)\left[P_{\tau_{k}}\left(p_{k} f\right)\right]\left(\zeta_{l, i}^{-1}\right) \frac{1}{J_{\zeta, l, i}\left(\zeta_{l, i}^{-1}\right)} \chi_{\zeta_{l, i}\left(I_{i}\right)}
\end{aligned}
$$




$$
\begin{aligned}
= & \sum_{k=1}^{K} \sum_{l=1}^{L} \sum_{j=1}^{q} \sum_{i=1}^{q} r_{l}\left(\zeta_{l, i}^{-1}\right) p_{k}\left(\tau_{k, j}^{-1} \circ \zeta_{l, i}^{-1}\right) f\left(\tau_{k, j}^{-1} \circ \zeta_{l, i}^{-1}\right) \\
& \times \frac{1}{J_{\tau, k, j}\left(\tau_{k, j}^{-1} \circ \zeta_{l, i}^{-1}\right)} \frac{1}{J_{\zeta, l, i}\left(\zeta_{l, i}^{-1}\right)} \chi_{\tau_{k}\left(I_{j}\right)}\left(\zeta_{l, i}^{-1}\right) \chi_{\zeta_{l, i}\left(I_{i}\right)} \\
= & \sum_{k=1}^{K} \sum_{l=1}^{L} P_{\tau_{k} \circ \zeta_{l}}\left(p_{k}\left(\zeta_{l}\right) r_{l} f\right)=P_{T \circ R} f .
\end{aligned}
$$

4. The existence of an absolutely continuous invariant measure on $[a, b]$. Let $(I, \mathfrak{B}, \lambda)$ be a measure space, where $\lambda$ is normalized Lebesgue measure on $I=[a, b]$. Let $\tau_{k}: I \rightarrow I, k=1, \ldots, K$, be piecewise one-toone and differentiable, non-singular transformations on a partition $\mathcal{P}$ of $I$ : $\mathcal{P}=\left\{I_{1}, \ldots, I_{q}\right\}$ and $\tau_{k, i}=\left.\tau_{k}\right|_{I_{i}}, i=1, \ldots, q, k=1, \ldots, K$. Denote by $V(\cdot)$ the standard one-dimensional variation of a function, and by $\mathrm{BV}(I)$ the space of functions of bounded variation on $I$ equipped with the norm $\|\cdot\|_{\mathrm{BV}}=V(\cdot)+\|\cdot\|_{1}$.

Let $g_{k}(x)=p_{k}(x) /\left|\tau_{k}^{\prime}(x)\right|, k=1, \ldots, K$. We assume the following conditions:

Condition (A). $\sum_{k=1}^{K} g_{k}(x)<\alpha<1, x \in I$.

Condition (B). $g_{k} \in \mathrm{BV}(I), k=1, \ldots, K$.

Under the above conditions our goal is to prove

$$
V_{I} P_{T}^{n} f \leq A V_{I} f+B\|f\|_{1}
$$

for some $n \geq 1$, where $0<A<1$ and $B>0$. The inequality (4.1) guarantees the existence of a $T$-invariant measure absolutely continuous with respect to Lebesgue measure and the quasi-compactness of the operator $P_{T}$ with all the consequences of this fact (see [1]). We will need a number of lemmas:

Lemma 4.1. Let $f \in \mathrm{BV}(I)$. Suppose $\tau: I \rightarrow J$ is differentiable and $\tau^{\prime}(x) \neq 0, x \in I$. Set $\phi=\tau^{-1}$ and let $g(x)=p(x) /\left|\tau^{\prime}(x)\right| \in \operatorname{BV}(I)$. Then

$$
V_{J}(f(\phi) g(\phi)) \leq\left(V_{I} f+\sup _{I} f\right)\left(V_{I} g+\sup _{I} g\right) .
$$

Proof. First, note that we have dropped all the $k, i$ indices to simplify the notation. The proof follows in the same way as in Lemma 3 of [9].

Lemma 4.2. Let $T$ satisfy conditions (A) and (B). Then for any $f \in$ $\mathrm{BV}(I)$,

$$
V_{I} P_{T} f \leq A V_{I} f+B\|f\|_{1}
$$


where

$$
A=3 \alpha+\max _{1 \leq i \leq q} \sum_{k=1}^{K} V_{I_{i}} g_{k}, \quad B=2 \beta \alpha+\beta \max _{1 \leq i \leq q} \sum_{k=1}^{K} V_{I_{i}} g_{k}
$$

with $\beta=\max _{1 \leq i \leq q}\left(\lambda\left(I_{i}\right)\right)^{-1}$.

Proof. First, we will refine the partition $\mathcal{P}$ to satisfy an additional condition. Let $\eta>0$ be such that $\sum_{k=1}^{K}\left(g_{k}(x)+\varepsilon_{k}\right)<\alpha$ whenever $\left|\varepsilon_{k}\right|<\eta$, $k=1, \ldots, K$. Since $g_{k}, k=1, \ldots, K$, are of bounded variation we can find a finite partition $\mathcal{K}$ such that for any $k=1, \ldots, K$,

$$
\left|g_{k}(x)-g_{k}(y)\right|<\eta
$$

for $x, y$ in the same element of $\mathcal{K}$. Without loss of generality, we can assume that our original partition is the join $\mathcal{P} \vee \mathcal{K}$. Then

$$
\max _{1 \leq i \leq q} \sum_{k=1}^{K} \sup _{x \in I_{i}} g_{k}(x)<\alpha \text {. }
$$

We have $V_{I}\left(P_{T} f\right)=V_{I}\left(\sum_{k=1}^{K} P_{\tau_{k}}\left(p_{k} f\right)\right)$. We will estimate this variation. Let $\phi_{k, i}=\tau_{k, i}^{-1}, k=1, \ldots, K, i=1, \ldots, q$. We have

$$
\begin{aligned}
V_{I}\left(\sum_{k=1}^{K} P_{\tau_{k}}\left(p_{k} f\right)\right)= & V_{I}\left(\sum_{k=1}^{K} \sum_{i=1}^{q} f\left(\phi_{k, i}\right) g_{k}\left(\phi_{k, i}\right) \chi_{\tau_{k}\left(I_{i}\right)}\right) \\
\leq & \sum_{k=1}^{K} \sum_{i=1}^{q}\left[\left|f\left(a_{i-1}\right)\right|\left|g_{k}\left(a_{i-1}\right)\right|+\left|f\left(a_{i}\right)\right|\left|g_{k}\left(a_{i}\right)\right|\right] \\
& +\sum_{k=1}^{K} \sum_{i=1}^{q} V_{\tau_{k}\left(I_{i}\right)}\left[f\left(\phi_{k, i}\right) g_{k}\left(\phi_{k, i}\right)\right]
\end{aligned}
$$

First, we estimate the first sum on the right hand side of (4.2):

$$
\begin{aligned}
\sum_{k=1}^{K} \sum_{i=1}^{q} & {\left[\left|f\left(a_{i-1}\right)\right|\left|g_{k}\left(a_{i-1}\right)\right|+\left|f\left(a_{i}\right)\right| \| g_{k}\left(a_{i}\right) \mid\right] } \\
& =\sum_{i=1}^{q}\left[\left|f\left(a_{i-1}\right)\right|\left(\sum_{k=1}^{K}\left|g_{k}\left(a_{i-1}\right)\right|\right)+\left|f\left(a_{i}\right)\right|\left(\sum_{k=1}^{K}\left|g_{k}\left(a_{i}\right)\right|\right)\right] \\
& \leq \alpha\left(\sum_{i=1}^{q}\left(\left|f\left(a_{i-1}\right)\right|+\left|f\left(a_{i}\right)\right|\right)\right) \\
& \leq \alpha\left(\sum_{i=1}^{q}\left(V_{I_{i}} f+\left(\lambda\left(I_{i}\right)\right)^{-1} \int_{I_{i}} f d \lambda\right)\right)=\alpha\left(V_{I} f+\beta\|f\|_{1}\right) .
\end{aligned}
$$


We now estimate the second term on the right hand side of (4.2). Using Lemma 4.1 we obtain

$$
\begin{aligned}
\sum_{k=1}^{K} \sum_{i=1}^{q} V_{\tau_{k}\left(I_{i}\right)}\left[f\left(\phi_{k, i}\right) g_{k}\left(\phi_{k, i}\right)\right] & \\
\leq & \sum_{k=1}^{K} \sum_{i=1}^{q}\left(V_{I_{i}} f+\sup _{I_{i}} f\right)\left(V_{I_{i}} g_{k}+\sup _{I_{i}} g_{k}\right) \\
& \leq \sum_{i=1}^{q}\left(2 V_{I_{i}} f+\beta \int_{I_{i}} f d \lambda\right)\left(\max _{1 \leq i \leq q} \sum_{k=1}^{K}\left(V_{I_{i}} g_{k}+\sup _{I_{i}} g_{k}\right)\right) \\
& \leq\left(2 V_{I} f+\beta\|f\|_{1}\right)\left(\max _{1 \leq i \leq q} \sum_{k=1}^{K} V_{I_{i}} g_{k}+\alpha\right) .
\end{aligned}
$$

Thus, using (4.3) and (4.4), we obtain

$$
V_{I} P_{T} f \leq\left(3 \alpha+\max _{1 \leq i \leq q} \sum_{k=1}^{K} V_{I_{i}} g_{k}\right) V_{I} f+\left(2 \beta \alpha+\beta \max _{1 \leq i \leq q} \sum_{k=1}^{K} V_{I_{i}} g_{k}\right)\|f\|_{1} .
$$

In the following two lemmas we show that the constants $\alpha$ as well as $\max _{1 \leq i \leq q} \sum_{k=1}^{K} V_{I_{i}} g_{k}$ decrease when we consider higher iterations $T^{n}$ instead of $T$. The constant $\beta$ obviously increases, but this is not important.

Lemma 4.3. Let $T$ be a random map which satisfies condition (A). Then, for $x \in I$,

$$
\sum_{w \in\{1, \ldots, K\}^{N}} \frac{p_{w}(x)}{\left|T_{w}^{\prime}(x)\right|}<\alpha^{N}
$$

where $T_{w}(x)=\tau_{k_{N}} \circ \tau_{k_{N-1}} \circ \cdots \circ \tau_{k_{1}}(x)$ and $p_{w}(x)=p_{k_{N}}\left(\tau_{k_{N-1}} \circ \cdots \circ \tau_{k_{1}}(x)\right)$. $p_{k_{N-1}}\left(\tau_{k_{N-2}} \circ \cdots \circ \tau_{k_{1}}(x)\right) \cdots p_{k_{1}}(x)$ define the random map $T^{N}$.

Proof. We have

$$
T^{N}(x)=\tau_{k_{N}} \circ \tau_{k_{N-1}} \circ \cdots \circ \tau_{k_{1}}(x)
$$

with probability

$$
p_{k_{N}}\left(\tau_{k_{N-1}} \circ \cdots \circ \tau_{k_{1}}(x)\right) \cdot p_{k_{N-1}}\left(\tau_{k_{N-2}} \circ \cdots \circ \tau_{k_{1}}(x)\right) \cdots p_{k_{1}}(x) .
$$

The maps defining $T^{N}$ may be indexed by $w \in\{1, \ldots, K\}^{N}$. Set

$$
T_{w}(x)=\tau_{k_{N}} \circ \tau_{k_{N-1}} \circ \cdots \circ \tau_{k_{1}}(x),
$$

where $w=\left(k_{1}, \ldots, k_{N}\right)$, and

$$
p_{w}(x)=p_{k_{N}}\left(\tau_{k_{N-1}} \circ \cdots \circ \tau_{k_{1}}(x)\right) \cdot p_{k_{N-1}}\left(\tau_{k_{N-2}} \circ \cdots \circ \tau_{k_{1}}(x)\right) \cdots p_{k_{1}}(x) .
$$

Then

$$
T_{w}^{\prime}(x)=\tau_{k_{N}}^{\prime}\left(\tau_{k_{N-1}} \circ \cdots \circ \tau_{k_{1}}(x)\right) \tau_{k_{N-1}}^{\prime}\left(\tau_{k_{N-2}} \circ \cdots \circ \tau_{k_{1}}(x)\right) \cdots \tau_{k_{1}}^{\prime}(x) .
$$


Suppose that $T$ satisfies condition (A). We will prove (4.5) using induction on $N$. For $N=1$, we have

$$
\sum_{w \in\{1, \ldots, K\}} \frac{p_{w}(x)}{\left|T_{w}^{\prime}(x)\right|}<\alpha
$$

by condition (A). Assume (4.5) is true for $N-1$. Then

$$
\begin{aligned}
& \sum_{w \in\{1, \ldots, K\}^{N}} \frac{p_{w}(x)}{\left|T_{w}^{\prime}(x)\right|}=\sum_{\bar{w} \in\{1, \ldots, K\}^{N-1}} \sum_{k=1}^{K} \frac{p_{k}(x) p_{\bar{w}}\left(\tau_{k}(x)\right)}{\left|\tau_{k}^{\prime}(x)\right|\left|T_{\bar{w}}^{\prime}\left(\tau_{k}(x)\right)\right|} \\
& \leq\left(\sum_{k=1}^{K} \frac{p_{k}(x)}{\left|\tau_{k}^{\prime}(x)\right|}\right)\left(\sum_{\bar{w} \in\{1, \ldots, K\}^{N-1}} \frac{p_{\bar{w}}\left(\tau_{k}(x)\right)}{\left|T_{\bar{w}}^{\prime}\left(\tau_{k}(x)\right)\right|}\right)<\alpha \cdot \alpha^{N-1}=\alpha^{N} .
\end{aligned}
$$

Lemma 4.4. Let $g_{w}=p_{w} /\left|T_{w}^{\prime}\right|$, where $T_{w}$ and $p_{w}$ are defined in Lemma 4.3 and $w \in\{1, \ldots, K\}^{n}$. Define

$$
W_{1} \equiv \max _{1 \leq i \leq q} \sum_{k=1}^{K} V_{I_{i}} g_{k}, \quad W_{n} \equiv \max _{J \in \mathcal{P}^{(n)}} \sum_{w \in\{1, \ldots, K\}^{n}} V_{J} g_{w},
$$

where $\mathcal{P}^{(n)}$ is the common monotonicity partition for all $T_{w}$. Then, for all $n \geq 1$,

$$
W_{n} \leq n \alpha^{n-1} W_{1}
$$

where $\alpha$ is defined in condition (A).

Proof. We prove the lemma by induction on $n$. For $n=1$ the assertion is true by definition of $W_{n}$. Assume that it is true for $n$, i.e.,

$$
W_{n} \leq n \alpha^{n-1} W_{1} .
$$

Let $J \in \mathcal{P}^{(n+1)}$ and $x_{0}<x_{1}<\cdots<x_{l}$ be a sequence of points in $J$. Then

$$
\begin{aligned}
& \sum_{w} \sum_{j=0}^{l-1}\left|g_{w}\left(x_{j+1}\right)-g_{w}\left(x_{j}\right)\right|=\sum_{j=0}^{l-1} \sum_{w \in\{1, \ldots, K\}^{n+1}}\left|g_{w}\left(x_{j+1}\right)-g_{w}\left(x_{j}\right)\right| \\
& \leq \sum_{j=0}^{l-1} \sum_{\bar{w} \in\{1, \ldots, K\}^{n}} \sum_{k=1}^{K}\left|g_{\bar{w}}\left(\tau_{k}\left(x_{j+1}\right)\right) g_{k}\left(x_{j+1}\right)-g_{\bar{w}}\left(\tau_{k}\left(x_{j}\right)\right) g_{k}\left(x_{j}\right)\right| \\
& \leq \sum_{j=0}^{l-1} \sum_{\bar{w} \in\{1, \ldots, K\}^{n}} \sum_{k=1}^{K}\left|g_{\bar{w}}\left(\tau_{k}\left(x_{j+1}\right)\right) g_{k}\left(x_{j+1}\right)-g_{\bar{w}}\left(\tau_{k}\left(x_{j+1}\right)\right) g_{k}\left(x_{j}\right)\right| \\
&+\sum_{j=0}^{l-1} \sum_{\bar{w} \in\{1, \ldots, K\}^{n}} \sum_{k=1}^{K}\left|g_{\bar{w}}\left(\tau_{k}\left(x_{j+1}\right)\right) g_{k}\left(x_{j}\right)-g_{\bar{w}}\left(\tau_{k}\left(x_{j}\right)\right) g_{k}\left(x_{j}\right)\right|
\end{aligned}
$$




$$
\begin{aligned}
\leq & \sum_{j=0}^{l-1} \sum_{k=1}^{K}\left|g_{k}\left(x_{j+1}\right)-g_{k}\left(x_{j}\right)\right| \sum_{\bar{w} \in\{1, \ldots, K\}^{n}} g_{\bar{w}}\left(\tau_{k}\left(x_{j+1}\right)\right) \\
& +\sum_{j=0}^{l-1} \sum_{k=1}^{K} g_{k}\left(x_{j}\right) \sum_{\bar{w} \in\{1, \ldots, K\}^{n}}\left|g_{\bar{w}}\left(\tau_{k}\left(x_{j+1}\right)\right)-g_{\bar{w}}\left(\tau_{k}\left(x_{j}\right)\right)\right| \\
\leq & \alpha^{n} \sum_{j=0}^{l-1} \sum_{k=1}^{K}\left|g_{k}\left(x_{j+1}\right)-g_{k}\left(x_{j}\right)\right| \\
& +\alpha \sum_{j=0}^{l-1} \sum_{\bar{w} \in\{1, \ldots, K\}^{n}}\left|g_{\bar{w}}\left(\tau_{k}\left(x_{j+1}\right)\right)-g_{\bar{w}}\left(\tau_{k}\left(x_{j}\right)\right)\right| \\
\leq & \alpha^{n} W_{1}+\alpha W_{n} \leq \alpha^{n} W_{1}+n \alpha^{n} W_{1}=(n+1) \alpha^{n} W_{1} .
\end{aligned}
$$

We used condition (A) and Lemma 4.3.

TheOREM 4.5. Let $T$ be a random map which satisfies conditions (A) and (B). Then $T$ preserves a measure which is absolutely continuous with respect to Lebesgue measure. The operator $P_{T}$ is quasi-compact on $\mathrm{BV}(I)$ (see [1]).

Proof. Let $N$ be such that $A_{N}=3 \alpha^{N}+W_{N}<1$. Then, by Lemma 4.3,

$$
\sum_{w \in\{1, \ldots, K\}^{N}} g_{w}(x)<\alpha^{N}, \quad x \in I .
$$

We refine the partition $\mathcal{P}^{(N)}$ as in the proof of Lemma 4.2, to have

$$
\max _{J \in \mathcal{P}^{N}} \sum_{w \in\{1, \ldots, K\}^{N}} \sup _{J} g_{w}<\alpha^{N} .
$$

Then, by Lemma 4.2, we get

$$
\left\|P_{T}^{N} f\right\|_{\mathrm{BV}} \leq A_{N}\|f\|_{\mathrm{BV}}+B_{N}\|f\|_{1},
$$

where $B_{N}=\beta_{N}\left(2 \alpha^{N}+W_{N}\right), \beta_{N}=\max _{J \in \mathcal{P}^{N}}(\lambda(J))^{-1}$. The theorem follows by the standard technique (see [1]).

REMARK 4.6. It is enough to assume that condition (A) is satisfied for some iterate $T^{m}, m \geq 1$.

REMARK 4.7. The number of absolutely continuous invariant measures for random maps has been studied in [4]. The proof of [4], which uses graph theoretic methods, goes through analogously in our case, i.e., when $T$ is a random map with position dependent probabilities. 
5. Example. We present an example of a random map $T$ which does not satisfy the conditions of [5], yet it preserves an absolutely continuous invariant measure under conditions (A) and (B).

EXAMPLE 5.1. Let $T$ be a random map which is given by $\left\{\tau_{1}, \tau_{2} ; p_{1}(x)\right.$, $\left.p_{2}(x)\right\}$, where

$$
\begin{aligned}
& \tau_{1}(x)= \begin{cases}2 x \quad \text { for } 0 \leq x \leq 1 / 2, \\
x \quad \text { for } 1 / 2<x \leq 1,\end{cases} \\
& \tau_{2}(x)= \begin{cases}x+1 / 2 & \text { for } 0 \leq x \leq 1 / 2, \\
2 x-1 & \text { for } 1 / 2<x \leq 1,\end{cases}
\end{aligned}
$$

Then $\sum_{k=1}^{2} g_{k}(x)=2 / 3<1$. Therefore, $T$ satisfies conditions (A) and (B). Consequently, by Theorem 4.5, $T$ preserves an invariant measure absolutely continuous with respect to Lebesgue measure. Notice that $\tau_{1}, \tau_{2}$ are piecewise linear Markov maps defined on the same Markov partition $\mathcal{P}$ : $\{[0,1 / 2],[1 / 2,1]\}$. For such maps the Perron-Frobenius operator reduces to a matrix (see [1]). The corresponding matrices are:

$$
P_{\tau_{1}}=\left(\begin{array}{cc}
1 / 2 & 1 / 2 \\
0 & 1
\end{array}\right), \quad P_{\tau_{2}}=\left(\begin{array}{cc}
0 & 1 \\
1 / 2 & 1 / 2
\end{array}\right) .
$$

Their invariant densities are $f_{\tau_{1}}=[0,2]$ and $f_{\tau_{2}}=[2 / 3,4 / 3]$. The PerronFrobenius operator of the random map $T$ is given by

$$
P_{T}=\left(\begin{array}{cc}
2 / 3 & 0 \\
0 & 1 / 3
\end{array}\right)\left(\begin{array}{cc}
1 / 2 & 1 / 2 \\
0 & 1
\end{array}\right)+\left(\begin{array}{cc}
1 / 3 & 0 \\
0 & 2 / 3
\end{array}\right)\left(\begin{array}{cc}
0 & 1 \\
1 / 2 & 1 / 2
\end{array}\right)=\left(\begin{array}{ll}
1 / 3 & 2 / 3 \\
1 / 3 & 2 / 3
\end{array}\right) .
$$

If the invariant density of $T$ is $f=\left[f_{1}, f_{2}\right]$, normalized by $f_{1}+f_{2}=2$ and satisfying the equation $f P_{T}=f$, then $f_{1}=2 / 3$ and $f_{2}=4 / 3$.

6. The existence of an absolutely continuous invariant measure in $\mathbb{R}^{n}$. Let $S$ be a bounded region in $\mathbb{R}^{n}$ and $\lambda_{n}$ be Lebesgue measure on $S$. Let $\tau_{k}: S \rightarrow S, k=1, \ldots, K$, be piecewise one-to-one and $C^{2}$, non-singular transformations on a partition $\mathcal{P}$ of $S, \mathcal{P}=\left\{S_{1}, \ldots, S_{q}\right\}$ and $\tau_{k, i}=\left.\tau_{k}\right|_{S_{i}}$, $i=1, \ldots, q, k=1, \ldots, K$. Suppose each $S_{i}$ is a bounded closed domain having a piecewise $C^{2}$ boundary of finite $(n-1)$-dimensional measure. We assume that the faces of $\partial S_{i}$ meet at angles bounded uniformly away from 0 . We will also assume that the probabilities $p_{k}(x)$ are piecewise $C^{1}$ functions on the partition $\mathcal{P}$. Let $D \tau_{k, i}^{-1}(x)$ be the derivative matrix of $\tau_{k, i}^{-1}$ at $x$. We assume: 
Condition (C).

$$
\max _{1 \leq i \leq q} \sum_{k=1}^{K} p_{k}(x)\left\|D \tau_{k, i}^{-1}\left(\tau_{k, i}(x)\right)\right\|<\sigma<1 .
$$

Let $\sup _{x \in \tau_{k, i}\left(S_{i}\right)}\left\|D \tau_{k, i}^{-1}(x)\right\|=: \sigma_{k, i}$ and $\sup _{x \in S_{i}} p_{k}(x)=: \pi_{k, i}$. Using the smoothness of $D \tau_{k, i}^{-1}$ 's and $p_{k}$ 's we can refine the partition $\mathcal{P}$ to satisfy

Condition $\left(\mathrm{C}^{\prime}\right)$.

$$
\sum_{k=1}^{K} \max _{1 \leq i \leq q} \sigma_{k, i} \pi_{k, i}<\sigma<1 .
$$

Under this condition, our goal is to prove the existence of an a.c.i.m. for the random map $T=\left\{\tau_{1}, \ldots, \tau_{K} ; p_{1}, \ldots, p_{K}\right\}$. The main tool of this section is the multidimensional notion of variation defined using derivatives in the distributional sense (see [3]):

$$
V(f)=\int_{\mathbb{R}^{n}}\|D f\|=\sup \left\{\int_{\mathbb{R}^{n}} f \operatorname{div}(g) d \lambda_{n}: g=\left(g_{1}, \ldots, g_{n}\right) \in C_{0}^{1}\left(\mathbb{R}^{n}, \mathbb{R}^{n}\right)\right\},
$$

where $f \in L_{1}\left(\mathbb{R}^{n}\right)$ has bounded support, $D f$ denotes the gradient of $f$ in the distributional sense, and $C_{0}^{1}\left(\mathbb{R}^{n}, \mathbb{R}^{n}\right)$ is the space of continuously differentiable functions from $\mathbb{R}^{n}$ into $\mathbb{R}^{n}$ having a compact support. We will use the following property of variation which is derived from $[3$, Remark 2.14]: If $f=0$ outside a closed domain $A$ whose boundary is Lipschitz continuous, $\left.f\right|_{A}$ is continuous, $\left.f\right|_{\operatorname{int}(A)}$ is $C^{1}$, then

$$
V(f)=\int_{\operatorname{int}(A)}\|D f\| d \lambda_{n}+\int_{\partial A}|f| d \lambda_{n-1}
$$

where $\lambda_{n-1}$ is the $n$-1-dimensional measure on the boundary of $A$. In this section we shall consider the Banach space (see [3, Remark 1.12])

$$
\mathrm{BV}(S)=\left\{f \in L_{1}(S): V(f)<\infty\right\},
$$

with the norm $\|f\|_{\mathrm{BV}}=V(f)+\|f\|_{1}$. We adapt the following two lemmas from [6]. Their proofs are exactly the same as in [6].

Lemma 6.1. Consider $S_{i} \in \mathcal{P}$. Let $x$ be a point in $\partial S_{i}$ and $y=\tau_{k}(x)$ a point in $\partial\left(\tau_{k}\left(S_{i}\right)\right)$. Let $J_{k, i}$ be the Jacobian of $\left.\tau_{k}\right|_{S_{i}}$ at $x$ and $J_{k, i}^{0}$ be the Jacobian of $\left.\tau_{k}\right|_{\partial S_{i}}$ at $x$. Then

$$
\frac{J_{k, i}^{0}}{J_{k, i}} \leq \sigma_{k, i}
$$

Fix $1 \leq i \leq q$. Let $Z$ denote the set of singular points of $\partial S_{i}$. Let us construct for any $x \in Z$ the largest cone with vertex at $x$ and which lies 
completely in $S_{i}$. Let $\theta(x)$ denote the vertex angle of this cone. Then define

$$
\beta\left(S_{i}\right)=\min _{x \in Z} \theta(x) .
$$

Since the faces of $\partial S_{i}$ meet at angles bounded away from 0 , we have $\beta\left(S_{i}\right)$ $>0$. Let $\alpha\left(S_{i}\right)=\pi / 2+\beta\left(S_{i}\right)$ and

$$
a\left(S_{i}\right)=\left|\cos \left(\alpha\left(S_{i}\right)\right)\right| \text {. }
$$

Now we will construct a $C^{1}$ field of segments $L_{y}, y \in \partial S_{i}$, every $L_{y}$ being a central ray of a regular cone contained in $S_{i}$, with vertex angle at $y$ greater than or equal to $\beta\left(S_{i}\right)$.

We start at points $y \in Z$ where the minimal angle $\beta\left(S_{i}\right)$ is attained, defining $L_{y}$ to be central rays of the largest regular cones contained in $S_{i}$. Then we extend this field of segments to the $C^{1}$ field we want, making $L_{y}$ short enough to avoid overlapping. Let $\delta(y)$ be the length of $L_{y}, y \in \partial S_{i}$. By the compactness of $\partial S_{i}$ we have

$$
\delta\left(S_{i}\right)=\inf _{y \in \partial S_{i}} \delta(y)>0 .
$$

Now, we shorten the $L_{y}$ of our field, making them all of length $\delta\left(S_{i}\right)$.

Lemma 6.2. For any $S_{i}, i=1, \ldots, q$, if $f$ is a $C^{1}$ function on $S_{i}$, then

$$
\int_{\partial S_{i}} f(y) d \lambda_{n-1}(y) \leq \frac{1}{a\left(S_{i}\right)}\left(\frac{1}{\delta\left(S_{i}\right)} \int_{S_{i}} f d \lambda_{n}+V_{\operatorname{int}\left(S_{i}\right)}(f)\right) .
$$

Our main technical result is the following:

THeOREM 6.3. If $T$ is a random map which satisfies condition $(\mathrm{C})$, then

$$
V\left(P_{T} f\right) \leq \sigma(1+1 / a) V(f)+\left(M+\frac{\sigma}{a \delta}\right)\|f\|_{1},
$$

where $a=\min \left\{a\left(S_{i}\right): i=1, \ldots, q\right\}>0, \delta=\min \left\{\delta\left(S_{i}\right): i=1, \ldots, q\right\}>0$, and

$$
M_{k, i}=\sup _{x \in S_{i}}\left(D p_{k}(x)-\frac{D J_{k, i}}{J_{k, i}} p_{k}(x)\right), \quad M=\sum_{k=1}^{K} \max _{1 \leq i \leq q} M_{k, i} .
$$
let

Proof. We have $V\left(P_{T} f\right) \leq \sum_{k=1}^{K} V\left(P_{\tau_{k}}\left(p_{k} f\right)\right)$. To estimate $V\left(P_{\tau_{k}}\left(p_{k} f\right)\right)$,

$$
F_{k, i}=\frac{f\left(\tau_{k, i}^{-1}\right) p_{k}\left(\tau_{k, i}^{-1}\right)}{J_{k, i}\left(\tau_{k, i}^{-1}\right)}, \quad R_{k, i}=\tau_{k, i}\left(S_{i}\right), \quad i=1, \ldots, q, k=1, \ldots, K .
$$

Then

$$
\begin{aligned}
\int_{\mathbb{R}^{n}}\left\|D P_{\tau_{k}}\left(p_{k} f\right)\right\| d \lambda_{n} & \leq \sum_{i=1}^{q} \int_{\mathbb{R}^{n}}\left\|D\left(F_{k, i} \chi_{R_{i}}\right)\right\| d \lambda_{n} \\
& \leq \sum_{i=1}^{q}\left(\int_{\mathbb{R}^{n}}\left\|D\left(F_{k, i}\right) \chi_{R_{i}}\right\| d \lambda_{n}+\int_{\mathbb{R}^{n}}\left\|F_{k, i}\left(D \chi_{R_{i}}\right)\right\| d \lambda_{n}\right) .
\end{aligned}
$$


Now, for the first integral we have

$$
\begin{aligned}
& \int_{\mathbb{R}^{n}}\left\|D\left(F_{k, i}\right) \chi_{R_{i}}\right\| d \lambda_{n}=\int_{R_{i}}\left\|D\left(F_{k, i} p_{k}\right)\right\| d \lambda_{n} \\
& \leq \int_{R_{i}}\left\|D\left(f\left(\tau_{k, i}^{-1}\right)\right) \frac{p_{k}\left(\tau_{k, i}^{-1}\right)}{J_{k, i}\left(\tau_{k, i}^{-1}\right)}\right\| d \lambda_{n}+\int_{R_{i}}\left\|f\left(\tau_{k, i}^{-1}\right) D\left(\frac{p_{k}\left(\tau_{k, i}^{-1}\right)}{J_{k, i}\left(\tau_{k, i}^{-1}\right)}\right)\right\| d \lambda_{n} \\
& \leq \int_{R_{i}}\left\|D f\left(\tau_{k, i}^{-1}\right)\right\|\left\|D \tau_{k, i}^{-1}\right\| \frac{p_{k}\left(\tau_{k, i}^{-1}\right)}{J_{k, i}\left(\tau_{k, i}^{-1}\right)} d \lambda_{n}+\int_{R_{i}}\left\|f\left(\tau_{k, i}^{-1}\right)\right\| \frac{M_{k}}{J_{k, i}\left(\tau_{k, i}^{-1}\right)} d \lambda_{n} \\
& \leq \sigma_{k, i} \pi_{k, i} \int_{S_{i}}\|D f\| d \lambda_{n}+M_{k} \int_{S_{i}}\|f\| d \lambda_{n} .
\end{aligned}
$$

For the second integral we have

$$
\left.\int_{\mathbb{R}^{n}}\left\|F_{k, i}\left(D \chi_{R_{i}}\right)\right\| d \lambda_{n}=\int_{\partial R_{i}} \mid f\left(\tau_{k, i}^{-1}\right)\right)\left|\frac{p_{k}\left(\tau_{k, i}^{-1}\right)}{J_{k, i}\left(\tau_{k, i}^{-1}\right)} d \lambda_{n-1}=\int_{\partial S_{i}}\right| f \mid p_{k} \frac{J_{k, i}^{0}}{J_{k, i}} d \lambda_{n-1} .
$$

By Lemma 4.3, $J_{k, i}^{0} / J_{k, i} \leq \sigma_{k, i}$. Using Lemma 4.2, we get

$$
\begin{aligned}
\int_{\mathbb{R}^{n}}\left\|F_{k, i}\left(D \chi_{R_{i}}\right)\right\| d \lambda_{n} & \leq \sigma_{k, i} \pi_{k, i} \int_{\partial S_{i}}|f| d \lambda_{n-1} \\
& \leq \frac{\sigma_{k, i} \pi_{k, i}}{a} V_{S_{i}}(f)+\frac{\sigma_{k, i} \pi_{k, i}}{a \delta} \int_{S_{i}}|f| d \lambda_{n} .
\end{aligned}
$$

Using Condition $\left(\mathrm{C}^{\prime}\right)$, summing first over $i$, we obtain

$$
\begin{aligned}
V\left(P_{\tau_{k}}\left(p_{k} f\right)\right) \leq & \left(\max _{1 \leq i \leq q} \sigma_{k, i} \pi_{k, i}\right)(1+1 / a) V(f) \\
& +\left(\max _{1 \leq i \leq q} M_{k, i}+\frac{\max _{1 \leq i \leq q} \sigma_{k, i} \pi_{k, i}}{a \delta}\right)\|f\|_{1} .
\end{aligned}
$$

Then summing over $k$ yields the assertion.

TheOREM 6.4. Let $T$ be a random map which satisfies condition (C). If $\sigma(1+1 / a)<1$, then $T$ preserves a measure which is absolutely continuous with respect to Lebesgue measure. The operator $P_{T}$ is quasi-compact on $\mathrm{BV}(S)$ (see [1]).

Proof. This follows by the standard technique (see [1]).

7. Example in $\mathbb{R}^{2}$. In this section, we present an example of a random map which satisfies condition (C) of Theorem 6.3 and thus it preserves an absolutely continuous invariant measure. 
EXAMPLE 7.1. Let $T$ be a random map given by $\left\{\tau_{1}, \tau_{2} ; p_{1}(x), p_{2}(x)\right\}$, where $\tau_{1}, \tau_{2}: I^{2} \rightarrow I^{2}$ are defined by

$$
\begin{aligned}
& \tau_{1}\left(x_{1}, x_{2}\right) \\
& = \begin{cases}\left(3 x_{1}, 2 x_{2}\right) & \text { for }\left(x_{1}, x_{2}\right) \in S_{1}=\left\{0 \leq x_{1}, x_{2} \leq 1 / 3\right\} \\
\left(3 x_{1}-1,2 x_{2}\right) & \text { for }\left(x_{1}, x_{2}\right) \in S_{2}=\left\{1 / 3<x_{1} \leq 2 / 3 ; 0 \leq x_{2} \leq 1 / 3\right\}, \\
\left(3 x_{1}-2,2 x_{2}\right) & \text { for }\left(x_{1}, x_{2}\right) \in S_{3}=\left\{2 / 3<x_{1} \leq 1 ; 0 \leq x_{2} \leq 1 / 3\right\} \\
\left(3 x_{1}, 3 x_{2}-1\right) & \text { for }\left(x_{1}, x_{2}\right) \in S_{4}=\left\{0<x_{1} \leq 1 / 3 ; 1 / 3<x_{2} \leq 2 / 3\right\}, \\
\left(3 x_{1}-1,3 x_{2}-1\right) & \text { for }\left(x_{1}, x_{2}\right) \in S_{5}=\left\{1 / 3<x_{1}, x_{2} \leq 2 / 3\right\} \\
\left(3 x_{1}-2,3 x_{2}-1\right) & \text { for }\left(x_{1}, x_{2}\right) \in S_{6}=\left\{2 / 3<x_{1} \leq 1 ; 1 / 3<x_{2} \leq 2 / 3\right\}, \\
\left(3 x_{1}, 3 x_{2}-2\right) & \text { for }\left(x_{1}, x_{2}\right) \in S_{7}=\left\{0 \leq x_{1} \leq 1 / 3 ; 2 / 3<x_{2} \leq 1\right\}, \\
\left(3 x_{1}-1,3 x_{2}-2\right) & \text { for }\left(x_{1}, x_{2}\right) \in S_{8}=\left\{1 / 3<x_{1} \leq 2 / 3 ; 2 / 3<x_{2} \leq 1\right\}, \\
\left(3 x_{1}-2,3 x_{2}-2\right) & \text { for }\left(x_{1}, x_{2}\right) \in S_{9}=\left\{2 / 3<x_{1} \leq 1 ; 2 / 3<x_{2} \leq 1\right\},\end{cases}
\end{aligned}
$$

$$
\tau_{2}\left(x_{1}, x_{2}\right)= \begin{cases}\left(3 x_{1}, 3 x_{2}\right) & \text { for }\left(x_{1}, x_{2}\right) \in S_{1}, \\ \left(2-3 x_{1}, 3 x_{2}\right) & \text { for }\left(x_{1}, x_{2}\right) \in S_{2} \\ \left(3 x_{1}-2,3 x_{2}\right) & \text { for }\left(x_{1}, x_{2}\right) \in S_{3} \\ \left(3 x_{1}, 3 x_{2}-1\right) & \text { for }\left(x_{1}, x_{2}\right) \in S_{4}, \\ \left(2-3 x_{1}, 3 x_{2}-1\right) & \text { for }\left(x_{1}, x_{2}\right) \in S_{5} \\ \left(3 x_{1}-2,3 x_{2}-1\right) & \text { for }\left(x_{1}, x_{2}\right) \in S_{6} \\ \left(3 x_{1}, 3 x_{2}-2\right) & \text { for }\left(x_{1}, x_{2}\right) \in S_{7} \\ \left(2-3 x_{1}, 3 x_{2}-2\right) & \text { for }\left(x_{1}, x_{2}\right) \in S_{8} \\ \left(3 x_{1}-2,3 x_{2}-2\right) & \text { for }\left(x_{1}, x_{2}\right) \in S_{9}\end{cases}
$$

and

$$
p_{1}(x)=\left\{\begin{array}{ll}
0.215 & \text { for }\left(x_{1}, x_{2}\right) \in S_{1}, \\
0.216 & \text { for }\left(x_{1}, x_{2}\right) \in S_{2}, \\
0.216 & \text { for }\left(x_{1}, x_{2}\right) \in S_{3}, \\
0.216 & \text { for }\left(x_{1}, x_{2}\right) \in S_{4}, \\
0.215 & \text { for }\left(x_{1}, x_{2}\right) \in S_{5}, \\
0.216 & \text { for }\left(x_{1}, x_{2}\right) \in S_{6}, \\
0.216 & \text { for }\left(x_{1}, x_{2}\right) \in S_{7}, \\
0.216 & \text { for }\left(x_{1}, x_{2}\right) \in S_{8}, \\
0.215 & \text { for }\left(x_{1}, x_{2}\right) \in S_{9},
\end{array} \quad p_{2}(x)=\left\{\begin{array}{lll}
0.785 & \text { for }\left(x_{1}, x_{2}\right) \in S_{1}, \\
0.784 & \text { for }\left(x_{1}, x_{2}\right) \in S_{2}, \\
0.784 & \text { for }\left(x_{1}, x_{2}\right) \in S_{3}, \\
0.784 & \text { for }\left(x_{1}, x_{2}\right) \in S_{4}, \\
0.785 & \text { for }\left(x_{1}, x_{2}\right) \in S_{5}, \\
0.784 & \text { for }\left(x_{1}, x_{2}\right) \in S_{6}, \\
0.784 & \text { for }\left(x_{1}, x_{2}\right) \in S_{7}, \\
0.784 & \text { for }\left(x_{1}, x_{2}\right) \in S_{8}, \\
0.785 & \text { for }\left(x_{1}, x_{2}\right) \in S_{9} .
\end{array}\right.\right.
$$

The derivative matrix of $\tau_{1, i}^{-1}$ is

$$
\left(\begin{array}{cc}
1 / 3 & 0 \\
0 & 1 / 3
\end{array}\right) \quad \text { or } \quad\left(\begin{array}{cc}
1 / 3 & 0 \\
0 & 1 / 2
\end{array}\right)
$$


and the derivative matrix of $\tau_{2, i}^{-1}$ is

$$
\left(\begin{array}{cc}
1 / 3 & 0 \\
0 & 1 / 3
\end{array}\right) \quad \text { or } \quad\left(\begin{array}{cc}
-1 / 3 & 0 \\
0 & 1 / 3
\end{array}\right)
$$

Therefore, the Euclidean matrix norm $\left\|D \tau_{1, i}^{-1}\right\|$ is $\sqrt{2} / 3$ or $\sqrt{13} / 6$, and the Euclidean matrix norm $\left\|D \tau_{2, i}^{-1}\right\|$ is $\sqrt{2} / 3$. Then

$$
\max _{1 \leq i \leq q} \sum_{k=1}^{K} p_{k}(x)\left\|D \tau_{k, i}^{-1}\left(\tau_{k, i}(x)\right)\right\| \leq 0.216 \frac{\sqrt{13}}{6}+0.785 \frac{\sqrt{2}}{3} .
$$

For this partition $\mathcal{P}$, we have $a=1$, which implies

$$
\sigma(1+1 / a)=2\left(0.216 \frac{\sqrt{13}}{6}+0.785 \frac{\sqrt{2}}{3}\right) \approx 0.9998<1 .
$$

Therefore, by Theorem 6.4, the random map $T$ admits an absolutely continuous invariant measure. Notice that $\tau_{1}, \tau_{2}$ are piecewise linear Markov maps defined on the same Markov partition $\mathcal{P}=\left\{S_{1}, \ldots, S_{9}\right\}$. For such maps the Perron-Frobenius operator reduces to a matrix and the invariant density is constant on the elements of the partition (see [1]). The Perron-Frobenius operator of $T$ is represented by the matrix

$$
M=\Pi_{1} M_{1}+\Pi_{2} M_{2}
$$

where $M_{1}, M_{2}$ are the matrices of $P_{\tau_{1}}$ and $P_{\tau_{2}}$ respectively, and $\Pi_{1}, \Pi_{2}$ are the diagonal matrices of $p_{1}(x)$ and $p_{2}(x)$ respectively. Then $M$ is given by

$$
M=p_{1} \mathbf{I d}_{9} \cdot\left(\begin{array}{ccccccccc}
1 / 6 & 1 / 6 & 1 / 6 & 1 / 6 & 1 / 6 & 1 / 6 & 0 & 0 & 0 \\
1 / 6 & 1 / 6 & 1 / 6 & 1 / 6 & 1 / 6 & 1 / 6 & 0 & 0 & 0 \\
1 / 6 & 1 / 6 & 1 / 6 & 1 / 6 & 1 / 6 & 1 / 6 & 0 & 0 & 0 \\
1 / 9 & 1 / 9 & 1 / 9 & 1 / 9 & 1 / 9 & 1 / 9 & 1 / 9 & 1 / 9 & 1 / 9 \\
1 / 9 & 1 / 9 & 1 / 9 & 1 / 9 & 1 / 9 & 1 / 9 & 1 / 9 & 1 / 9 & 1 / 9 \\
1 / 9 & 1 / 9 & 1 / 9 & 1 / 9 & 1 / 9 & 1 / 9 & 1 / 9 & 1 / 9 & 1 / 9 \\
1 / 9 & 1 / 9 & 1 / 9 & 1 / 9 & 1 / 9 & 1 / 9 & 1 / 9 & 1 / 9 & 1 / 9 \\
1 / 9 & 1 / 9 & 1 / 9 & 1 / 9 & 1 / 9 & 1 / 9 & 1 / 9 & 1 / 9 & 1 / 9 \\
1 / 9 & 1 / 9 & 1 / 9 & 1 / 9 & 1 / 9 & 1 / 9 & 1 / 9 & 1 / 9 & 1 / 9
\end{array}\right)
$$




$$
+p_{2} \mathbf{I d}_{9} \cdot\left(\begin{array}{ccccccccc}
1 / 9 & 1 / 9 & 1 / 9 & 1 / 9 & 1 / 9 & 1 / 9 & 1 / 9 & 1 / 9 & 1 / 9 \\
1 / 9 & 1 / 9 & 1 / 9 & 1 / 9 & 1 / 9 & 1 / 9 & 1 / 9 & 1 / 9 & 1 / 9 \\
1 / 9 & 1 / 9 & 1 / 9 & 1 / 9 & 1 / 9 & 1 / 9 & 1 / 9 & 1 / 9 & 1 / 9 \\
1 / 9 & 1 / 9 & 1 / 9 & 1 / 9 & 1 / 9 & 1 / 9 & 1 / 9 & 1 / 9 & 1 / 9 \\
1 / 9 & 1 / 9 & 1 / 9 & 1 / 9 & 1 / 9 & 1 / 9 & 1 / 9 & 1 / 9 & 1 / 9 \\
1 / 9 & 1 / 9 & 1 / 9 & 1 / 9 & 1 / 9 & 1 / 9 & 1 / 9 & 1 / 9 & 1 / 9 \\
1 / 9 & 1 / 9 & 1 / 9 & 1 / 9 & 1 / 9 & 1 / 9 & 1 / 9 & 1 / 9 & 1 / 9 \\
1 / 9 & 1 / 9 & 1 / 9 & 1 / 9 & 1 / 9 & 1 / 9 & 1 / 9 & 1 / 9 & 1 / 9 \\
1 / 9 & 1 / 9 & 1 / 9 & 1 / 9 & 1 / 9 & 1 / 9 & 1 / 9 & 1 / 9 & 1 / 9
\end{array}\right)
$$

where

$$
\begin{aligned}
& p_{1}=(0.215,0.216,0.216,0.216,0.215,0.216,0.216,0.216,0.215), \\
& p_{2}=(0.785,0.784,0.784,0.784,0.785,0.784,0.784,0.784,0.785)
\end{aligned}
$$

Id $_{9}$ is the $9 \times 9$ identity matrix and

$$
\begin{gathered}
a=0.12306, \quad b=0.087222, \quad c=0.12311, \\
d=0.087111, \quad e=0.11111 .
\end{gathered}
$$

The invariant density of $T$ is

$$
f=\left(f_{1}, f_{2}, f_{3}, f_{4}, f_{5}, f_{6}, f_{7}, f_{8}, f_{9}\right), \quad f_{i}=f_{\mid S_{i}}, \quad i=1, \ldots, 9,
$$

normalized by

$$
f_{1}+f_{2}+f_{3}+f_{4}+f_{5}+f_{6}+f_{7}+f_{8}+f_{9}=9
$$

and satisfying the equation $f M=f$. Then

$$
f_{1}=f_{2}=f_{3}=f_{4}=f_{5}=f_{6}=\frac{9}{6.29739}, \quad f_{7}=f_{8}=f_{9}=\frac{0.29739}{3} f_{1} .
$$




\section{References}

[1] A. Boyarsky and P. Góra, Laws of Chaos, Birkhäuser, Boston, 1997.

[2] N. Dunford and J. T. Schwartz, Linear Operators, Part I: General Theory, Interscience, New York, 1964.

[3] E. Giusti, Minimal Surfaces and Functions of Bounded Variation, Birkhaüser, Boston, 1984.

[4] P. Góra, Graph-theoretic bound on number of a.c.i.m. for random transformations, Proc. Amer. Math. Soc. 116 (1992), 401-410.

[5] P. Góra and A. Boyarsky, Absolutely continuous invariant measures for random maps with position dependent probabilities, J. Math. Anal. Appl. 278 (2003), 225242.

[6] -, - Absolutely continuous invariant measures for piecewise expanding $C^{2}$ transformations in $\mathbb{R}^{N}$, Israel J. Math. 67 (1989), 272-286.

[7] Y. Kifer, Ergodic Theory of Random Transformations, Birkhäuser, Boston, 1986.

[8] T. Morita, Random iteration of one-dimensional transformations, Osaka J. Math. 22 (1985), 489-518.

[9] S. Pelikan, Invariant densities for random maps of the interval, Trans. Amer. Math. Soc. 281 (1984), 813-825.

[10] Ö. Stenflo, Uniqueness of invariant measures for place-dependent random iterations of functions, in: IMA Vol. Math. Appl. 132, Springer, 2002, 13-32.

Department of Mathematics and Statistics

University of Victoria

PO BOX 3045 STN CSC

Victoria, B.C., V8W 3P4, Canada

E-mail: wab@math.uvic.ca
Department of Mathematics and Statistics Concordia University 7141 Sherbrooke Street West Montreal, Quebec H4B 1R6, Canada E-mail: pgora@vax2.concordia.ca

Received December 19, 2003

Revised version August 23, 2004 